\title{
Propagação vegetativa de hibisco com diferentes tipos de estacas e concentrações de ácido indolbutírico
}

\author{
Rafaela Ribeiro de Souza ${ }^{1 *}$, Márkilla Zunete Beckmann Cavalcante², \\ Marluce Pereira Damasceno Lima', Tamnata Ferreira Alixandre', Rejane Teixeira Nascimento'
}

'Universidade Federal do Piauí, Campus Prof. ${ }^{a}$ CinobelinaElvas", Bom Jesus, Pl, Brasil

2Universidade Federal do Vale do São Francisco, Petrolina, PE, Brasil

*Autor correspondente, e-mail: rafaela_o2@hotmail.com

\section{Resumo}

A propagação do hibisco é feita preferencialmente por estaquia. Entretanto, o sucesso obtido nessa técnica depende dascondiçõesinternas e externas da estaca, que influenciarão diretamente em sua capacidade de enraizamento. Nesse sentido, objetivou-se avaliar o efeito de três tipos de estacas (apical, mediana e basal) e quatro concentrações de AIB (0, 1000, 3000 e $5000 \mathrm{mg} \mathrm{L}^{-1}$ ) na propagação via estaquia de Hibiscus rosa-sinensis L. cv. Snow Queen. O experimento foi conduzido em casa de vegetação sob nebulização intermitente na Universidade Federal do Piauí (UFPI), Campus Professora CinobelinaElvas, Bom Jesus-PI, no período de 23 de maio a 07 de julho de 2012. O delineamento experimental utilizado foi em blocos completamente casualizados, arranjados em esquema fatorial 3×4 (Tipo de estaca x Concentração de AIB) com quatro repetições. Aos 45 dias após a implantação do experimento foram avaliados: porcentagem de enraizamento, taxa de sobrevivência, número de brotos, número de folhas, comprimento médio da raiz, massa seca da parte aérea e massa seca de raiz. Não houve efeito significativo da interação entre os fatores estudados, havendo efeito apenas para tipo de estaca. A técnica de propagação via estaquia de Hibiscus rosa-sinensis L. cv. Snow Queen é eficiente mediante ao uso de estacas basais.

Palavras-chave: auxinas, enraizamento, Hibiscus rosa-sinensisL.

\section{Vegetative Propagation of hibiscus with different types of cuttings and IBA concentrations}

\begin{abstract}
The propagation of the hibiscus is done preferably by cutting. However, the success of this technique depends on the internal and external conditions cuttings, that will influence directly into your rooting capacity. In this sense, the objective was to evaluate the effect of three types of cuttings (apical, middle and basal) and four concentrations of IBA (0, 1000, 3000 and $\left.5000 \mathrm{mg} \mathrm{L}^{-1}\right)$ in the propagation through cuttings of Hibiscus rosa-sinensisL. CV. Snow Queen. The experiment was conducted in a greenhouse under intermittent mist at the Federal University of Piauí (UFPI), Campus Profa. CinobelinaElvas, Bom Jesus-PI for the period from May 23 to July 7, 2012. The experimental design was completely randomized in a factorial arrangement $3 \times 4$ (Type of cuttings $x$ Concentration IBA) with four replications. At 45 days after implantation of the experiment were evaluated: percentage of rooting, survival rate, number of shoots, number of leaves, average root length, dry weight of shoot and root dry weight. There was no significant interaction between the factors studied. There is effective for cutting type. The technique of propagation by cuttings of Hibiscus rosa-sinensis L. CV. Snow Queen is efficient through the use of basal cuttings.
\end{abstract}

Keywords: auxins, rooting, Hibiscus rosa-sinensis L. 


\section{Introdução}

O Hibisco pertencente à família das Malvaceas éconhecido popularmente por papoula hibisco ou graxa-de-estudante. É um arbusto lenhoso, originário da Ásia tropical e do Havaí, que pode atingir até cinco metros de altura. Trata-se de uma espécie que apresenta floração durante todo o ano com flores de diversos formatos e cores(Lorerenzi, 2008). Os principais métodos de propagação para produção de mudas dessa espécie são por enxertia, alporquia e estaquia. Entre os métodos de propagação vegetativa citados, a estaquia é a de maior viabilidade por ser uma técnica simples, de baixo custo, além de apresentar rapidez na obtenção das mudas, precocidade e uniformidade (Hartmann et al., 2002; Contessa et al., 2011).

Para Denaxa et al. (2012), o sucesso na propagação por estaquia depende da distribuição de hormônios, da condição fisiológica e nutricional da planta matriz, do hábito de crescimento da planta (herbáceo ou lenhoso) e das condições ambientais (substratos, luminosidade, temperatura e umidade relativa do ar). Entretanto, tem-se observado que a condição fisiológica e a posição no ramo de onde se obtém as estacas são fatores preponderantes ao enraizamento. Pois, ao longo do ramo ocorrem variações nos conteúdos de reservas e substâncias promotoras e inibidoras do crescimento (Rapaka et al., 2005; Guo et al., 2009; Zerche\&Druege, 2009).

A formação de raiz adventícia é controlada por hormônios, e a aplicação exógena de reguladores vegetais em plantas, tais como auxinas e citocininas, é frequentemente utilizada na propagação vegetativa objetivando melhorar o enraizamento e a qualidade de transplantio (Balestri et al., 2012). No entanto, Ludwig-Müller (2011) afirma que a formação de raiz adventícia está diretamente conectada a ação das auxinas, pois elas são encarregadas de estimular a divisão celular e o processo de indução ao enraizamento é dependente de sua presença.

A resposta à aplicação exógena das auxinas varia de acordo com a espécie, ambiente e condições fisiológicas de cada estaca. Muitos estudos têm sido realizados com diversas plantas ornamentais para avaliar o comportamento de diferentes espécies, cultivares e tipos de estacas ás diferentes concentrações de auxinas (Pizzatto et al. (2011); Amaral et al. (2012); Pereira et al. (2012)).

Diante do exposto, o objetivo deste trabalho foi avaliar o potencial de enraizamento de estacas de Hibiscus rosa-sinensisL. cv. Snow Queen, em função do tipo de estaca e aplicação exógena de ácido indolbutírico (AIB).

\section{Material e Métodos}

O experimento foi realizado com estacas de Hibiscus rosa-sinensis L. cv. Snow Queen, no Setor de Horticultura do Campus Profa. CinobelinaElvas (CPCE) da Universidade Federal do Piauí (UFPI) situado no município de Bom

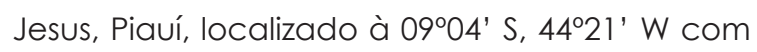
altitude média de $277 \mathrm{~m}$. Os dados climáticos referentes à temperatura e umidade relativa do ar foram obtidos diariamente com o auxílio de termo-higrômetro digital (Quimis ${ }^{\circledR}$ ) instalado na parte central da casa de vegetação, com o sensor disposto a 1,50 m de altura do solo.

O delineamento experimental adotado foi em blocos ao acaso com os tratamentos distribuídos em esquema fatorial $3 \times 4$, referentes a três tipos de estaca (posição apical, mediana e basal do ramo) e às quatro concentrações de ácido indolbutírico (AIB) (0, 1000, 3000 e 5000 $\mathrm{mg} \mathrm{L}^{-1}$ ) com quatro repetições e dez estacas por parcela, perfazendo um total de 480 estacas.

As estacas foram coletadas em plantas adultas de Hibiscus rosa-sinensisL. cv. Snow Queen, na região mediana da copa em pleno desenvolvimento vegetativo. A planta matriz nãoapresentava tratos culturais específicos quanto à adubação, poda, controle de pragas edoenças. Os ramos retirados da planta matriz foram dispostos provisoriamente em um recipiente com água, para evitar a desidratação e oxidação. Em seguida, para obtenção dos diferentes tipos de estacas, os ramos foram divididos em três segmentos: apical, mediano e basal.

As estacas foram padronizadas com no mínimo duas gemas, comprimentos de aproximadamente 7,10 e $12 \mathrm{~cm}$ e diâmetros de 
2, 4 e $6 \mathrm{~mm}$ para estacas apicais, medianas e basais respectivamente. Nas estacas basais e medianas deixou-se um par de folhas cortadas ao meio e, nas apicais, um par de folhas inteiras, as demais foram eliminadas.

A aplicação do AIB lácido indol-3butírico, Merck $^{\circledR}$, Alemanha), foi realizada na forma de pó, seguindo metodologia descrita por HARTMANN et al. (2002). As estacas foram dispostas em caixas plásticas de $30 \mathrm{~cm} \times 44$ $\mathrm{cm} \times 7 \mathrm{~cm}$ (largura $\times$ comprimento $\times$ altura) contendo como substrato areia lavada, e mantidas sob telado com $50 \%$ de sombreamento sob nebulização intermitente. O tempo de nebulização adotado foi de 15 minutos espaçados de 45 minutos, controlados por um temporizador.

Aos 45 dias após o plantio, as estacas foram retiradas cuidadosamente das bandejas, lavadas em água corrente e, avaliadas quanto à porcentagem de estacas enraizadas (EE); taxa de sobrevivência das estacas (SE); número de brotos por estaca (NBE); número de folhas (NF); comprimento médio da raiz (CMR) e massas seca da parte aérea (MSPA) e raiz (MSR).

Os dados obtidos foram submetidos à análise de variância para diagnóstico de efeito significativo em tipo de estacas e concentração de AIB. Quando o efeito para tipos de estacas foi significativo, as médias foram comparadas pelo teste de Tukey $(p<0,05)$. As análises foram realizadas pelo programa computacional Sistema para Análise de Variância - SISVAR, (Ferreira, 2011), sendo os dados transformados em $\sqrt{x}+0,5 \quad$ (Equação 1)

\section{Resultados e Discussão}

Não foi observado efeito significativo da interação entre os diferentes tipos de estacas e concentrações de ácido indolbutírico (AIB) nas variáveis. Entretanto houve diferença significativa entre os níveis do fator tipo de estaca (basal, mediana e apical) em todas as variáveis avaliadas (Tabela 1).

Tabela 1. Resumo da análise de variância de Hibiscus rosa-sinensis L. cv. Snow Queen para tipos de estacas e doses de AIB: porcentagem de estacas enraizadas (EE), taxa de sobrevivência das estacas (SE), número de brotos da estaca (NBE), número de folhas (NF), Comprimento médio da raiz adventícia (CMR), massa seca da parte aérea (MSPA) e massa seca de raiz (MSR).

\begin{tabular}{cccccccc}
\hline Causa de Variância & EE (\%) & SE & NBE & NF & CMR & MSPA & MSR \\
& $-\%$ & $-\%-$ & - & - & $-\mathrm{cm}-$ & $-\mathrm{g}-$ & $-\mathrm{g}-$ \\
\hline Tipos de estacas ('F') & $38,26^{*}$ & $74,86^{*}$ & $63,07^{*}$ & $62,18^{*}$ & $29,16^{*}$ & $65,84^{*}$ & $23,30^{*}$ \\
\hline Apical (A) & $3,3 \mathrm{~b}$ & $30 \mathrm{~b}$ & $0,50 \mathrm{c}$ & $1,41 \mathrm{C}$ & $0,04 \mathrm{~b}$ & $0,01 \mathrm{c}$ & $0,00 \mathrm{~b}$ \\
Mediana (M) & $61 \mathrm{a}$ & $95 \mathrm{a}$ & $1,66 \mathrm{~b}$ & $7,75 \mathrm{~b}$ & $4,37 \mathrm{a}$ & $0,12 \mathrm{~b}$ & $0,01 \mathrm{~b}$ \\
Basal (B) & $75 \mathrm{a}$ & $100 \mathrm{a}$ & $3,14 \mathrm{a}$ & $14,23 \mathrm{a}$ & $6,29 \mathrm{a}$ & $0,32 \mathrm{a}$ & $0,04 \mathrm{a}$ \\
DMS & 27 & 15,9 & 0,75 & 3,66 & 2,66 & 0,08 & 0,02 \\
\hline Doses de IBA ('F') & $0,45 \mathrm{~ns}$ & $0,28 \mathrm{~ns}$ & $0,68 \mathrm{~ns}$ & $1,85 \mathrm{~ns}$ & $1,37 \mathrm{~ns}$ & $1,80 \mathrm{~ns}$ & $1,55 \mathrm{~ns}$ \\
\hline IBA x tipos de estaca('F') & $0,28 \mathrm{~ns}$ & $1,10 \mathrm{~ns}$ & $0,44 \mathrm{~ns}$ & $1,28 \mathrm{~ns}$ & $1,25 \mathrm{~ns}$ & $1,89 \mathrm{~ns}$ & $1,14 \mathrm{~ns}$ \\
\hline CV \% & 33,3 & 20,2 & 13,8 & 18,8 & 26,1 & 4,8 & 1,72 \\
\hline Médias seguidas de letras distintas na coluna diferem entre si, pelo teste Tukey (p<0,05): * significativo (p<0,05); ns - não significativo. & &
\end{tabular}

Os resultados obtidos neste experimento diferem dos obtidos por Pereira et al. (2012), que verificaram significância para o efeito da interação dos diferentes tipos de estacas e as concentrações de $\operatorname{AIB}\left(0,250,2000 \mathrm{mg} \mathrm{L}^{-1}\right)$ em relação ao percentual de tecido caloso, enraizamento e brotação em estacas de Alamanda (Allamanda cathartica L.), enquanto que Loneet al.(2010) trabalhando com estacas herbáceas de azaléia (Rhododendron thomsonii $\mathrm{H}$.) em diferentes concentrações de AIB (0 e $1000 \mathrm{mg} \mathrm{L}^{-1}$ ) e em diferentes substratos, não observaram efeito significativo para concentrações de AIB.
Ao avaliar a propagação vegetativa via estaquia de malvavisco (Malvaviscus arboreus Cav.) com diferentes tipos de estacas e concentrações de AIB, Loss et al. (2009) obtiveram efeito significativo entre diferentes tipos de estacas e concentrações de AIB, concluindo que o uso de estacas lenhosas de malvavisco com a utilização de AIB na concentração de 2000 mg L-1 aumenta o potencial de enraizamento, contribuindo para formação de mudas com melhor padrão de mercado.

As concentrações de AIB utilizadas neste experimento não apresentaram efeito 
significativo sobre o enraizamento das estacas (Tabela 1). A ausência de significância pode ser supostamente explicada pela presença de auxinas endógenas nas estacas em níveis suficientes para formação de raízes. O uso de reguladores vegetais em propagação via estaquia visa estabelecer um equilíbrio favorável ao enraizamento, portanto o balanço hormonal entre níveis de auxinas endógenas e exógenas é de grande importância para a formação de raízes. $O$ AlB apresenta uma taxa lenta de conjugação, de forma que o AIB livre, necessário à fase de indução ao enraizamento, será disponibilizado após um longo período de tempo (Osterc \& Stampar, 2011; Ludwig-Muller, 2011). Neste sentido, é possível que durante a fase de indução (isto é, a fase mais sensível à auxina) a auxina livre poderia estar indisponível.

Pizzato et al. (2011), objetivando avaliar o efeito de concentrações de AIB, tamanho da estaca e época de realização da propagação vegetativa via estaquia de hibisco, verificaram efeito significativo das concentrações de AIB. No entanto, estacas sem aplicação exógena do AlB, apresentaram enraizamento acima de $70 \%$. Relacionando esses resultados com os obtidos neste trabalho, é possivel afirmar que a propagação via estaquia de hibisco dispensa a aplicação exógena de AIB.

A porcentagem de enraizamento foi influenciada pelo tipo de estaca, obtendo-se valores médios para estacas apicais, medianas e basais de 3,3; 61 e 75\%, respectivamente (Tabela 1). De acordo com Fachinello et al. (2005) a diferença quanto a capacidade de enraizamento ao longo do ramo da planta pode ocorrer devido a variação existente na concentração de fitormônios. Neste sentido as maiores porcentagens de enraizamento ocorridas nas estacas basais também podem estar associadas a uma maior reserva de nutrientes que é um dos fatores responsáveis pela maior predisposição ao enraizamento adventício, visto que, estacas basais apresentam maior diâmetro (Zerche \& Druege, 2009).

Os resultados obtidos neste experimento diferem dos obtidos por Maia et al. (2008), que ao avaliar o enraizamento de estacas de alfazemade-caboclo (Hyptis suaveolens) em função da posição da estaca no ramo, verificaram menores percentagens de estacas enraizadas na posição basal, sugerindo que este material apresentava algum impedimento para a formação das raízes adventícias.

Taiz \& Zeiger (2008) afirmam que posições inferiores do ramo são menos favoráveis à diferenciação radicular, pois a região basal apresenta maior grau de lignificação e menor conteúdo de auxinas, sendo o ápice caulinar o principal local de síntese desses hormônios. Porém, o potencial de enraizamento pode depender também, da distribuição de hormônios, da condição fisiológica e nutricional da planta matriz e do hábito de crescimento da planta (herbáceo ou lenhoso), o que justifica a diversidade de respostas em diferentes espécies (Ehlert et al., 2004; Denaxa et al., 2012).

Quanto à taxa de sobrevivência, observou-se para estacas basais e medianas valores de $100 \%$ e $95 \%$ respectivamente, enquanto que estacas apicais apresentaram taxa de sobrevivência inferior a 50\% (Tabela 1). A consistência dasestacas apicais, associado às condições de elevadas temperaturas (média de $33,7^{\circ} \mathrm{C}$ ) e baixa umidade do ar (média de $27,6 \%$ ) durante a condução do experimento (Figura 1), podem ter favorecido uma maior perda de água pelas estacas, prejudicando o enraizamento e sobrevivência das mesmas. De acordo com Hartmann et al. (2002), a faixa média de temperatura ideal para o enraizamento de estacas da maioria das espécies é de $21,1^{\circ} \mathrm{C}$ a $26^{\circ} \mathrm{C}$, enquantoque a umidade do ar na região das estacas ideal é de $80 \%$ a $100 \%$, o que permite a manutenção da turgescência dos tecidos e sobrevivência das mesmas.

A temperatura e umidade relativa do ar são fatores importantes à propagação vegetativa via estaquia. Embora, temperaturas elevadas possam aumentar o metabolismo, estimulando a diferenciação das células e o desenvolvimento das raízes, pode também favorecer a perda de água pelas folhas. Quanto à umidade, é importante evitar níveis críticos, principalmente para estacas com folhas, pois, aumenta os riscos de desidratação das mesmas levando à morte antes que as raízes se formem (Hartmann et al., 2002; Zang et al., 2013). 
O maior número de brotações, assim como o maior número de folhas foram obtidos em estacas basais, ao passo que estacas apicais apresentaram menor número (Tabela 1). Esse resultado indica que as estacas apicais apresentaram interferência provocada pela dominância apical existente nesta região. As estacas que apresentaram maior comprimento médio de raízes foram às estacas basais seguida das medianas (Tabela 1), indicando que, para estacas de hibisco quanto mais próximo as estacas forem retiradas da base do ramo, maiores são as condições para brotação e emissão de raízes adventícias, possibilitando a obtenção de mudas com melhor qualidade.

A maior massa seca de raízes também foi verificada nas estacas da posição basal (Tabela 1), tal média foi elevada devido ao grande volume de raízes emitidas nas referidas estacas. Da mesma forma, para a massa seca da parte aérea, forte indicador do vigor da muda, observou-se maior acúmulo em estacas colhidas da posição basal, esse maior acúmulo possivelmente ocorreu em decorrência da rápida formação e desenvolvimento das raízes e consequentemente maior absorção e translocação de fotoassimilados para parte aérea.

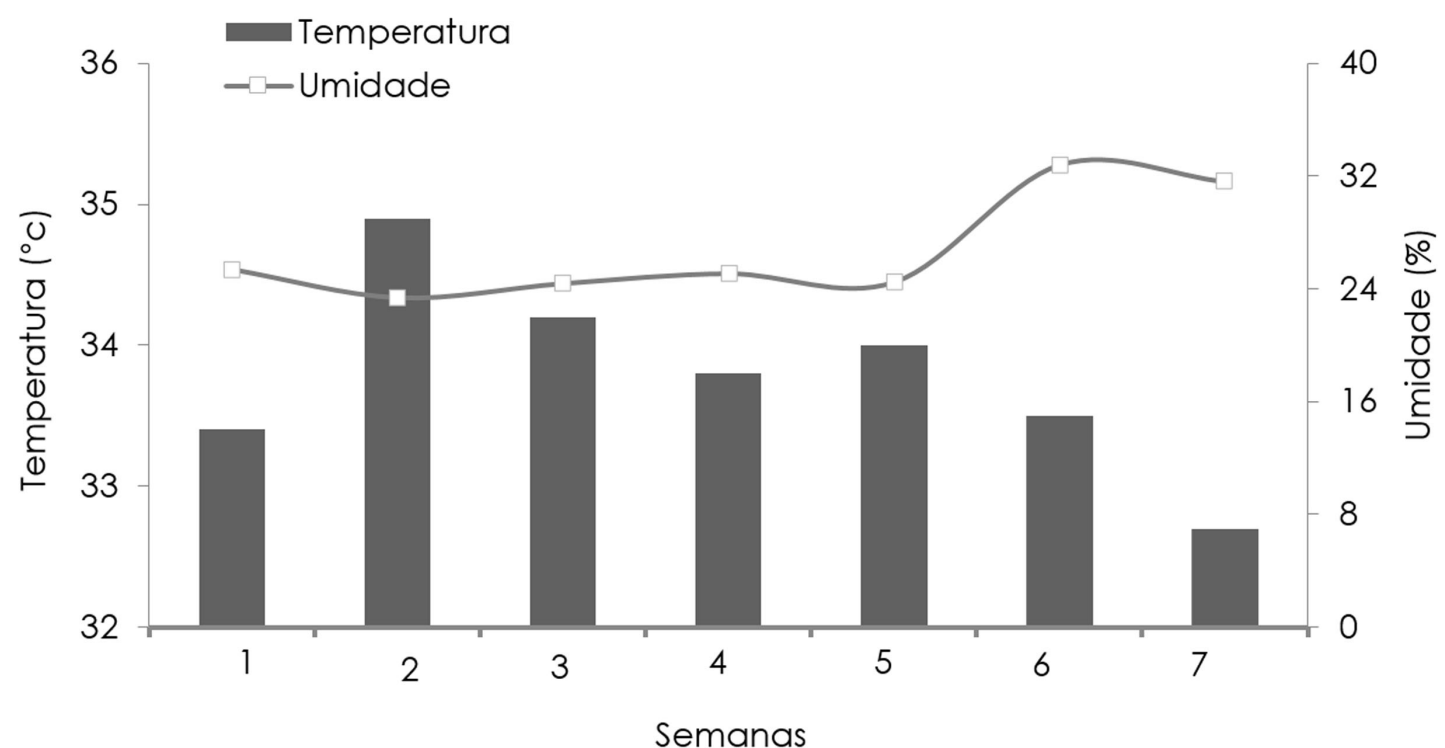

Figura 1. Médias referentes à temperatura $\left({ }^{\circ} \mathrm{C}\right)$ e umidade (\%) obtidas na casa de nebulização durante o período experimental, Bom Jesus, Estado do Piauí, 2012.

\section{Conclusões}

A propagação via estaquia de Hibiscus rosa-sinensis L. CV. Snow Queen é adequada mediante ao uso de estacas basais.

\section{Referências}

Amaral, G.C., Brito, L.P.S., Avelino, R.C., Júnior, J.V.S., Beckmann-Cavalcante, M.Z., Cavalcante, I.H.L. 2012. Produção de mudas de Durantarepens L. Revista de Ciências Agrárias 35: 134-142.

Balestri, E., Vallerini, F., Castelli, A., Lardicci, C. 2012. Application of plant growth regulators, a simple technique for improving the establishment success of plant cuttings in coastal dune restoration. Estuarine, Coastal and Shelf Science 99: 74-84.

Contessa, C., Valentini, N., Botta, R. 2011
Decreasing the concentration of IBA or combination with ethylene inhibitors improve bud retention in semi-hardwood cuttings of hazelnut cultivar 'TondaGentile delle Langhe'. Scientia Horticulturae 131: 103-106.

Denaxa, N.K., Vemmos, S.N., Roussos, P.A. 2012. The role of endogenous carbohydrates and seasonal variation in rooting ability of cuttings of an easy and a hard to root olive cultivars (Olea europaea L.). Scientia Horticulturae 143: 19-28.

Ehlert, P.A.D., Luz, J.M.Q., Innecco, R. 2004. Propagação vegetativa da alfavaca-cravo utilizando diferentes tipos de estacas e substratos. Horticultura Brasileira 22: 10-13.

Fachinello, J.C., Hoffmann, A.; Nachtigal, J.C. 2005. Propagação de plantas frutíferas.Embrapa, Brasília, Brasil. $221 \mathrm{p}$. 
Ferreira, D.F. 2011. Sisvar: A computer statistic alanalysis system. Ciência e Agrotecnologia 35: 1039-1042.

Guo, X.F., Fu, X.L., Zang, D.K., Ma, Y. 2009. Effect of auxin treatments, cuttings'collection date and initial characteristic son Paeonia'Yang Fei Chu Yu' cutting propagation. Scientia Horticulturae 119: 177-181.

Hartmann, H.T., Kester, D.E., Davies JR, F.T., Geneve, R.L.2002. Plant propagation: principles and practices. Prentice Hall International, New Jersey, USA. 770p.

Lone, A.B., Unemoto, L.K., Yamamoto, L.Y., Costa,L., Schnitzer, J. A., Sato, A.J., Ricce, W.S., Assis, A.M., Roberto, S.R. 2010. Enraizamento de estacas de azaleia (Rhododendron simsii Planch.) no outono em AIB e diferentes substratos. Ciência Rural 40: $1720-1725$.

Lorenzi, H. 2008. Plantas ornamentais no Brasil: arbustivas, herbáceas e trepadeiras. Instituto Plantarum, Nova Odessa, Brasil. 1130 p.

Loss, A., Teixeira, M.B., Santos, T.J., Gomes, V.M., Queiroz, L.H. 2009. Indução do enraizamento em estacas de Malvaviscus arboreus Cav. com diferentes concentrações de ácido indolbutírico (AIB). Acta Scientiarum Agronomy 31: 269-273.

Ludwig-Müller, J. 2011. Auxin conjugates: their role for plant development and in the evolution of land plants. Journalof Experimental Botany 62: 1757-1773.

Maia, S.S.S., Pinto, J.E.B.P., Silva, F.N., Oliveira, C.2008. Enraizamento de Hyptis suaveolens(L.) Poit. (Lamiaceae) em função da posição da estaca no ramo. Revista Brasileira de Ciências Agrárias 3: 317-320.

Osterc, G., Stampar, F. 2011. Differences in endo/ exogenous auxin prole in cuttings of different physiological ages. Journal of Plant Physiology 168: 2088-2092.

Pereira, G.H.A., Coutinho, F.S., Costa e Silva, R.A., Loss, A. 2012. Desenvolvimento de estacas de alamanda amarela sob diferentes concentrações de ácido indolbutírico. Comunicata Scientiae 3: 6-22.

Pizzato, M., Júnior, A.W., Luckmann, D., Pirola, K., Cassol, D.A., Mazaro, S.M. 2011 . Influência do uso de AIB, época de coleta e tamanho de estaca na propagação vegetativa de hibisco por estaquia. Revista Ceres 58: 487-492.

Rapaka, V.K., Bessler, B., Schreiner, M., Druege, U. 2005. Interplay between initial carbohydrate availability, current photosynthesis, and adventitious root formation in Pelargonium cuttings. Plant Science 168:1547-1560.

Taiz, L., Zeiger, E. 2009. Fisiologia Vegetal. Artmed, Porto Alegre, Brasil. 720p.

Zang, W., Song, L., Silva, JA.T., Sun, H. 2013. Effects of temperature, plant growth regulators and substrates andchanges in carbohydrate content during bulblet formation by twinscale propagation in Hippeastrum vittatum'Red lion'. Scientia Horticulturae 160: 230-237.

Zerche, S., Druege, U. 2009. Nitrogen content determines adventitious rooting in Euphorbia pulcherrima under adequate light independently of pre-rooting carbohydrate depletion of cuttings. Scientia Horticulturae 121: 340-347. 